\title{
Identification of colorectal cancer-restricted microRNAs and their target genes based on high-throughput sequencing data
}

This article was published in the following Dove Press journal:

OncoTargets and Therapy

24 March 2016

Number of times this article has been viewed

\section{Jing Chang \\ Liya Huang \\ Qing Cao \\ Fang Liu}

Department of Gerontology, Xinhua Hospital Affiliated to Jiaotong University School of Medicine, Shanghai, People's Republic of China

Correspondence: Fang Liu

Department of Gerontology, Xinhua Hospital Affiliated to Jiaotong University School of Medicine, No 1665, Kongjiang Road, Shanghai 200092,

People's Republic of China

Tel +8602125077733

Fax +8602165561819

Email fangliuflf@।63.com
Abstract: To identify potential key microRNAs (miRNAs) and their target genes for colorectal cancer (CRC). High-throughput sequencing data of miRNA expression and gene expression (ID: GSE46622) were downloaded from Gene Expression Omnibus, including matched colon tumor, normal colon epithelium, and liver metastasis tissues from eight CRC patients. Paired $t$-test and NOISeq separately were utilized to identify differentially expressed miRNAs (DE-miRNAs) and genes. Then, target genes with differential expression and opposite expression trends were identified for DE-miRNAs. Combined with tumor suppressor gene, tumor-associated gene, and TRANSFAC databases, CRC-restricted miRNAs were screened out based on miRNA-target pairs. Compared with normal tissues, there were 56 up- and 37 downregulated miRNAs in metastasis tissues, as well as eight up- and 30 downregulated miRNAs in tumor tissues. miRNA-1 was downregulated in tumor and metastasis tissues, while its target oncogenes TWIST1 and GATA4 were upregulated. Besides, miRNA-let-7f-1-3p was downregulated in tumor tissues, which also targeted TWIST1. In addition, miRNA-133b and miRNA-4458 were downregulated in tumor tissues, while their common target gene DUSP9 was upregulated. Conversely, miRNA-450b-3p was upregulated in metastasis tissues, while its target tumor suppressor gene CEACAM7 showed downregulation. The identified CRC-restricted miRNAs might be implicated in cancer progression via their target genes, suggesting their potential usage in CRC treatment.

Keywords: colorectal cancer, differentially expressed microRNAs, differentially expressed genes, oncogenes, tumor suppressor genes

\section{Introduction}

Colorectal cancer $(\mathrm{CRC})$ is the third most common malignancy cancer, causing 1,400,000 new cases and 694,000 deaths in 2012 worldwide. ${ }^{1}$ With great progresses in diagnosis and treatment, the overall survival of patients with early-stage CRC has been improved. However, in terms of the patients with advanced CRC, chemotherapy usually fails to achieve satisfactory therapeutic effects. ${ }^{2}$ Therefore, it is greatly important to further understand the molecular mechanism of CRC so as to discover novel therapeutic targets.

MicroRNAs (miRNAs) are a class of small noncoding RNAs participating in diverse biological processes such as cell differentiation, proliferation, and apoptosis through a myriad of targets. ${ }^{3}$ Importantly, miRNAs act as downstream regulators of driver genes or protein kinases in cancers, ${ }^{4}$ and targeting miRNAs is considered as a potential strategy to increase the specificity of therapy and to overcome drug resistance. Recently, a link between the aberrant expression of miRNAs and CRC progression has been reported. ${ }^{5,6}$ For instance, miRNA-21 has been demonstrated to be overexpressed in CRC, mediating tumor progression and cancer cell growth. ${ }^{7,8}$ On the contrary, miRNA-338-3p, 
miRNA-let-7, and miRNA-145 act as growth suppressors in CRC cells. ${ }^{9-11}$ In addition, downregulated miRNA-34a exerts suppressive effects on cell proliferation through regulating E2F signaling pathway in CRC. ${ }^{12}$ Serum miR-17-3p and miR-106a are biomarkers that can reflect tumor dynamics and predict disease recurrence for stage II/III CRC patients. ${ }^{13}$ Alteration in miRNA binding sites of cell envelope-associated proteinase genes, oculocutaneous albinism, and ocular albinism genes can inhibit translation process at genomic levels and disrupt cell cycle, which may in turn induce cancer or other diseases. ${ }^{14,15}$ Similarly, in silico evidence shows that single nucleotide polymorphisms in miRNA binding sites have effect on CRC. ${ }^{16,17}$ The miR-587/PPP2R1B/pAKT/XIAP signaling axis plays an essential role in regulating response to chemotherapy in CRC. ${ }^{18}$ Therefore, the identification of aberrantly expressed miRNAs and their targets is important to elucidate the initiation and progression of CRC.

Next-generation sequencing can help globally identify candidate miRNAs implicated in cancer. ${ }^{19}$ In 2013 , Röhr et $\mathrm{al}^{20}$ applied high-throughput sequencing for miRNAs and mRNAs from paired normal, tumor, and metastasis tissues to explore the therapeutic application of miRNA-1 in CRC. However, although Röhr et al ${ }^{20}$ have validated the great potential of miRNA-1 as therapeutic targets in CRC, there are still some important values to comprehensively and jointly analyze the miRNA and mRNA sequencing data using various bioinformatics methods.

In our study, we assumed that miRNA profile can be applied to predict clinical progression of CRC. To verify the hypothesis, bioinformatics approaches were first utilized to screen the differentially expressed miRNAs (DE-miRNAs) and genes (DEGs) in CRC based on the high-throughput data by Röhr et al. ${ }^{20}$ Thereafter, CRC-restricted miRNAs with differential expression were screened out according to the miRNAs-target pairs. The present findings revealed some new miRNAs implicated in CRC via their target genes, which can be used as novel therapeutic targets for CRC.

\section{Materials and methods}

\section{Data acquisition}

The high-throughput data GSE46622 deposited by Röhr et al ${ }^{20}$ were downloaded from the National Center of Biotechnology Information (NCBI) Gene Expression Omnibus (GEO, http://www.ncbi.nlm.nih.gov/geo/), ${ }^{21}$ which contained both miRNA and mRNA sequencing data based on the platform of Illumina Genome Analyzer IIx (Illumina, San Diego, CA, USA). For miRNA sequencing, a total of 24 samples, including the matched primary colon tumor tissues $(n=8)$, normal colon epithelium $(n=8)$, and liver metastasis tissues $(n=8)$ were collected from eight $\mathrm{CRC}$ patients undergoing surgical resection (mean age $=66.63$ years, six stable and two instable). In addition, GSE46622 also contained RNA-sequencing (RNA-seq) data of the paired samples collected from four of those patients. All samples were evaluated histopathologically and the required areas were macrodissected before RNA extraction. Then, RNA was extracted using the Trizol reagent (Invitrogen, Thermo Fisher Scientific, Waltham, MA, USA) according to the manufacturer's instructions. After smallRNA isolation and cDNA library preparation, RNA sequencing was performed by Illumina's RNA-Seq prep kit and Illumina's DGE smallRNA sample (Illumina). This study used microarray data downloaded from a public database, therefore, no ethics committee approval or patient consent were required.

\section{Data preprocessing}

The raw sequencing data were performed with quality control using FastQC (http://www.bioinformatics.bbsrc.ac.uk/ projects/fastqc). ${ }^{22}$ The reads containing either more than 50\% low quality bases $(<18)$ or over $3 \% \mathrm{~N}$ content in each end of the full lengths were filtered out. Besides, such noncoding RNAs, including rRNA, tRNA, and small nuclear RNA (snRNA) were eliminated based on reference sequences from rfam11.0 (http://rfam.xfam.org/). ${ }^{23}$ Afterward, the clean reads were aligned against the reference human genome hg19 (http://www.genome.ucsc.edu/index.html) using Bowtie 2 (http://computing.bio.cam.ac.uk/local/doc/bowtie2.html) ${ }^{24}$ and TopHat v1.3.3 (http://ccb.jhu.edu/software/tophat/index. $\underline{s h t m l})^{25}$ with default parameters. The sequences of mature miRNAs and pre-miRNAs were obtained from mirbase 20 (http://www.mirbase.org/) $)^{26}$ to identify miRNAs.

\section{Differential analysis of miRNAs}

MiRDeep2 (http://biowulf.nih.gov/apps/mirdeep2.html) ${ }^{27}$ emerges as a completely overhauled tool to predict miRNAs, which contains known and novel miRNAs in seven species. Herein, miRDeep2 was used to predict the novel miRNAs and calculate the expression levels of those known and predicted miRNAs. After that, paired $t$-test was used to identify DE-miRNAs in the following pairwise comparisons: tumor versus normal, metastasis versus tumor, and metastasis versus normal. The $P$-value $<0.05$ was selected as the cutoff criterion.

Clustering analysis was performed for the DE-miRNAs between normal and metastasis tissues. In addition, Venn diagrams were constructed for the up- and downregulated miRNAs in the aforementioned three comparisons, respectively. 


\section{Differential analysis of genes}

Cufflinks v2.1.1 (http://cufflinks.cbcb.umd.edu/index. $\underline{\mathrm{html}})^{28}$ was applied to calculate the gene expression levels, namely fragments per kilobase of exon per million fragments mapped values. Subsequently, NOISeq (http://www. bioconductor.org/packages/release/bioc/html/NOISeq. $\underline{\mathrm{html}})^{29}$ was used to screen DEGs in the following pairwise comparisons: tumor versus normal, metastasis versus tumor, and metastasis versus normal. The $q$-value $\geq 0.99$ was taken as the threshold.

\section{Screening of target genes for DE-miRNAs} miRNAs function through transcriptionally regulating expression levels of target genes in vivo. Thus, two kinds of databases were introduced to identify targets for DE-miRNAs, including predicted databases (miRanda, PITA, MirTarget2, PicTar, and TargetScan ${ }^{30}$ ), and validated databases (miRWalk ${ }^{31}$ and miRecords ${ }^{32}$ ). The genes, which appeared in no less than two of the predicted databases or in at least one of the validated databases, were screened out to be potential targets of DE-miRNAs.

\section{Mining of CRC-restricted miRNAs}

In the aforementioned analysis, target genes of DE-miRNAs were extracted according to the predicted and the validated databases. Then, the target genes were compared with the DEGs to identify differentially expressed targets. Following, differentially expressed targets were further mined to obtain targets with opposite expression trends with the miRNAs, termed as candidate targets of DE-miRNAs. Finally, the candidate targets of DE-miRNAs were input into tumor suppressor gene database, ${ }^{33}$ tumor-associated gene database, ${ }^{34}$ or TRANSFAC database ${ }^{35}$ to identify CRC-related candidate targets. Based on the pairs of miRNAs and targets, the
miRNAs corresponding to CRC-related candidate targets were selected as CRC-restricted miRNAs.

\section{Results \\ Screening of DE-miRNAs}

Compared with normal tissues, there separately were 56 upand 37 downregulated miRNAs in metastasis tissues, as well as eight up- and 30 downregulated miRNAs in tumor tissues (Table 1). Besides, there were 32 up- and one downregulated miRNAs in metastasis tissues relative to tumor tissues (Table 1). The number of upregulated miRNAs in metastasis tissues was more than that in tumor tissues, implying a more serious dysregulation in advanced colon cancer. Then, the cluster analysis was performed to explore the changes of the DE-miRNAs, indicating a clear separation between normal tissues and metastasis tissues (Figure 1). In addition, using MiRDeep2, a total of one, 18, and 24 upregulated novel miRNAs separately were identified in the following comparisons: tumor versus normal, metastasis versus tumor, and metastasis versus normal. On the other hand, two and six downregulated novel miRNAs were obtained from tumor and metastasis tissues in comparison to the normal tissues, respectively.

Based on the Venn diagram of upregulated miRNAs in the three comparisons (Figure 2A), a total of three common miRNAs, including miRNA-548ap-5p, miRNA-548j-5p, and miRNA-6850-5p were identified in tumor and metastasis tissues in comparison to normal tissues. A total of four common miRNAs, including miRNA-21-3p, miRNA-3648, miRNA4306, and miRNA-936 were found in the two comparisons (metastasis versus tumor and metastasis versus normal), suggesting persistent upregulation of those miRNAs in the three kinds of tissues. According to the Venn diagram of downregulated miRNAs in different comparisons (Figure 2B),

Table I Differentially expressed miRNAs in different comparisons (tumor vs normal, metastasis vs tumor, and metastasis vs normal)

\begin{tabular}{|c|c|c|c|}
\hline $\begin{array}{l}\text { Differentially } \\
\text { expressed miRNAs }\end{array}$ & Comparisons & Number & List of miRNAs \\
\hline \multirow[t]{3}{*}{$\begin{array}{l}\text { Upregulated } \\
\text { miRNAs }\end{array}$} & Tumor vs normal & 8 & $\begin{array}{l}\text { hsa-miR-548ap-5p, hsa-miR-548j-5p, hsa-miR-6850-5p, hsa-miR-503-3p, hsa-let-7a-5p, } \\
\text { hsa-miR-629-5p, etc }\end{array}$ \\
\hline & Metastasis vs tumor & 32 & hsa-miR-2I-3p, hsa-miR-3648, hsa-miR-4306, hsa-miR-936, etc \\
\hline & Metastasis vs normal & 56 & $\begin{array}{l}\text { hsa-miR-548ap-5p, hsa-miR-548j-5p, hsa-miR-6850-5p, hsa-miR-2I-3p, hsa-miR-3648, } \\
\text { hsa-miR-4306, hsa-miR-936, etc }\end{array}$ \\
\hline \multirow[t]{3}{*}{$\begin{array}{l}\text { Downregulated } \\
\text { miRNAs }\end{array}$} & Tumor vs normal & 30 & $\begin{array}{l}\text { hsa-miR-I, hsa-miR-I265, hsa-miR-I33a-3p, hsa-miR-133a-5p, hsa-miR-I33b, hsa-miR- } \\
\text { 338-3p, hsa-miR-497-5p, hsa-miR-605-3p, hsa-miR-6780a-5p, hsa-miR-433-3p, etc }\end{array}$ \\
\hline & Metastasis vs tumor & I & hsa-miR-548ab \\
\hline & Metastasis vs normal & 37 & $\begin{array}{l}\text { hsa-miR-I, hsa-miR-1265, hsa-miR-I33a-3p, hsa-miR-I33a-5p, hsa-miR-133b, hsa- } \\
\text { miR-338-3p, hsa-miR-497-5p, hsa-miR-605-3p, hsa-miR-548ai, hsa-miR-192-5p, etc }\end{array}$ \\
\hline
\end{tabular}

Note: Number, the number of up- or downregulated miRNAs in each comparison.

Abbreviations: miRNAs, microRNAs; vs, versus. 



Figure I Cluster heat map of the differentially expressed miRNAs in normal, tumor, and metastasis tissues.

Notes: The color scale indicates the relative expression levels of miRNAs; horizontal axis stands for samples (pln-p8n, eight normal tissues; plt-p8t, eight tumor tissues; $\mathrm{plm}-\mathrm{p} 8 \mathrm{~m}$, eight metastasis tissues); vertical coordinate represents differentially expressed miRNAs. The blue and green indicate that the differentially expressed miRNAs were divided into two clusters.

Abbreviation: miRNAs, microRNAs.

a total of eight common miRNAs (miRNA-1, miRNA-1265, miRNA-133a-3p, miRNA-133a-5p, miRNA-133b, miRNA338-3p, miRNA-497-5p, and miRNA-605-3p) were found in tumor and metastasis tissues compared with normal tissues.

\section{Screening of DEGs}

Relative to normal tissues, there separately were 1,552 upand 207 downregulated genes in tumor tissues, as well as
992 up- and 861 downregulated genes in metastasis tissues. In addition, there were 485 up- and 1,323 downregulated genes in metastasis tissues compared with tumor tissues (Table 2).

\section{Identification of CRC-restricted miRNAs}

To screen out the CRC-restricted miRNAs, the differentially expressed target genes were screened out and further
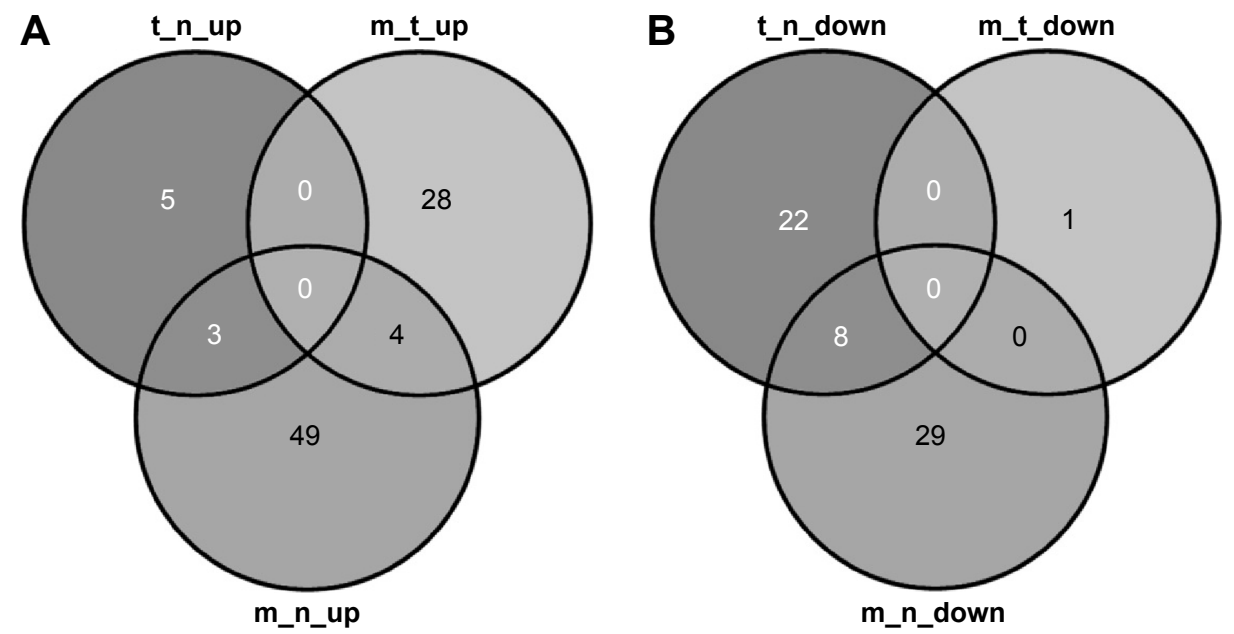

Figure 2 Venn diagrams of differentially expressed miRNAs in three comparisons (tumor vs normal, metastasis vs tumor, and metastasis vs normal).

Notes: (A) Venn diagrams of upregulated miRNAs. (B) Venn diagrams of downregulated miRNAs. In the diagram, $n, t$, and $m$ stand for normal, tumor, and metastasis samples, respectively.

Abbreviations: miRNAs, microRNAs; vs, versus. 
Table 2 Differentially expressed genes in different comparisons (tumor vs normal, metastasis vs tumor, and metastasis vs normal)

\begin{tabular}{lll}
\hline Comparisons & $\begin{array}{l}\text { Upregulated } \\
\text { genes }\end{array}$ & $\begin{array}{l}\text { Downregulated } \\
\text { genes }\end{array}$ \\
\hline Tumor vs normal & $\mathrm{I}, 552$ & 207 \\
Metastasis vs tumor & 485 & $\mathrm{I}, 323$ \\
Metastasis vs normal & 992 & $86 \mathrm{I}$ \\
\hline
\end{tabular}

Abbreviation: vs, versus.

mined to obtain those with opposite expression trends with the miRNAs. Accordingly, eligible target genes were identified for four up- and 12 downregulated miRNAs between tumor and normal tissues, as well as for 13 up- and 16 downregulated miRNAs between metastasis and normal tissues. Finally, the candidate targets of those DE-miRNAs were input into tumor suppressor gene database, tumor-associated gene database, or TRANSFAC database to identify CRC-related candidate targets. Based on the pairs of miRNAs and targets, the miRNAs corresponding to CRC-related candidate targets were selected as CRC-restricted miRNAs.

As shown in Figure 3, miRNA-1 was downregulated in both tumor and metastasis tissues relative to normal tissues, while its target oncogenes twist family bHLH transcription factor 1 (TWIST1) and GATA binding protein 4 (GATA4) were upregulated. In addition, miRNA-133b, miRNA-let7f-1-3p, miRNA-4458, and miRNA-338-3p were downregulated in tumor tissues and associated with CRC. Dual specificity phosphatase 9 (DUSP) was the common targets of miRNA-133b and miRNA-4458 and was upregulated in tumor tissues. Besides, miRNA-450b-3p was upregulated in metastasis tissues, and its target gene carcinoembryonic antigen (CEA)-related cell adhesion molecule 7 (CEACAM7) exhibited downregulation.

\section{Discussion}

In recent years, miRNAs have been identified not only as diagnostic and prognostic molecules but also as underlying therapeutic targets. In the current study, by analyzing matched normal, tumor, and metastasis tissues of eight CRC patients, we found that miRNA-1 and miRNA-let-7f-1-3p were downregulated, while their common target TWIST1 was upregulated. Besides, miRNA-133b and miRNA-4458 were found to be downregulated in tumor tissues, while their common target DUSP exhibited upregulation.

Quantitative real-time polymerase chain reaction (PCR) shows that miRNA-1 is downregulated, and it can mediate proliferation in CRC by targeting oncogene c-met (MET). ${ }^{36}$ Our results were in accordance with previous study that the expression level of miRNA-1 was lower in CRC.
Especially, miRNA-let-7f-1-3p was also downregulated in the current study, whose correlation with CRC has not been recorded in previous literatures. However, miRNAlet-7 has already been considered as a growth suppressor in CRC cells, ${ }^{9}$ suggesting the potential involvement of miRNA-let-7f-1-3p in CRC. Moreover, as miRNAs act through their target genes, we identified a list of DEGs as the potential target genes of miRNA-1 and miRNAlet-7f-1-3p. Among those target genes, downregulated TWIST1 was commonly targeted by miRNA-let-7f-1-3p and miRNA-1. As previously reported, TWIST1, a highly conserved transcription factor, involves in the metastasis via the regulation of epithelial-to-mesenchymal transition. ${ }^{37}$ The aberrant expression of TWIST1 can lead to the loss of cell-cell adhesion ${ }^{38}$ that may further cause the arrest of G2/M phase as well as apoptosis in CRC cells. ${ }^{39}$ Real-time PCR declares that expression level of TWIST1 mRNA is upregulated, thus, TWIST1 has been suggested to be a potential marker of poor outcome in CRC patients. ${ }^{40}$ Consistently, upregulation of TWIST1 in our analysis further confirmed that TWIST1 might be a therapeutic target for inhibiting the progression of CRC. As TWIST1 was targeted by miRNA-let-7f-1-3p and miRNA-1, we inferred that miRNA-1 and miRNA-let-7f-1-3p might be considered as therapeutic targets to inhibit the initial and development of CRC.

Meanwhile, miRNA-133b and miRNA-4458 were also found to be downregulated in tumor tissues in our analysis. The downregulation of miRNA-133b in bladder cancer has been revealed by real-time $\mathrm{PCR}^{41}$ that might inhibit cell growth and invasion in the progression of cancer via targeting Fascin homolog 1 gene $(F S C N 1){ }^{42}$ At present, no published reports demonstrate the involvement of miRNA4458 in cancer. In this study, we screened out a common target for miRNA-133b and miRNA-4458, namely overexpressed DUSP9. DUSP9 is a member of protein tyrosine phosphatases family important for controlling cell growth and cell survival in tumorigenesis. ${ }^{43}$ DUSP9 belongs to DUSPs identified as the negative regulators of MAPKs, ${ }^{44}$ which play crucial roles in CD147-promoted invasion and epithelial-tomesenchymal transition of CRC cells. ${ }^{45}$ A strong expression of DUSP9 has been demonstrated in the early stages of CRC in a mouse model, ${ }^{46}$ we thus inferred that miRNA-133b and miRNA-4458 targeting DUSP9 might be used as novel targets for CRC treatment.

Conversely, the level of miRNA-450b-3p was identified to be overexpressed in metastasis tissues in our study, which has never been reported in CRC. CEACAM7 was the target 


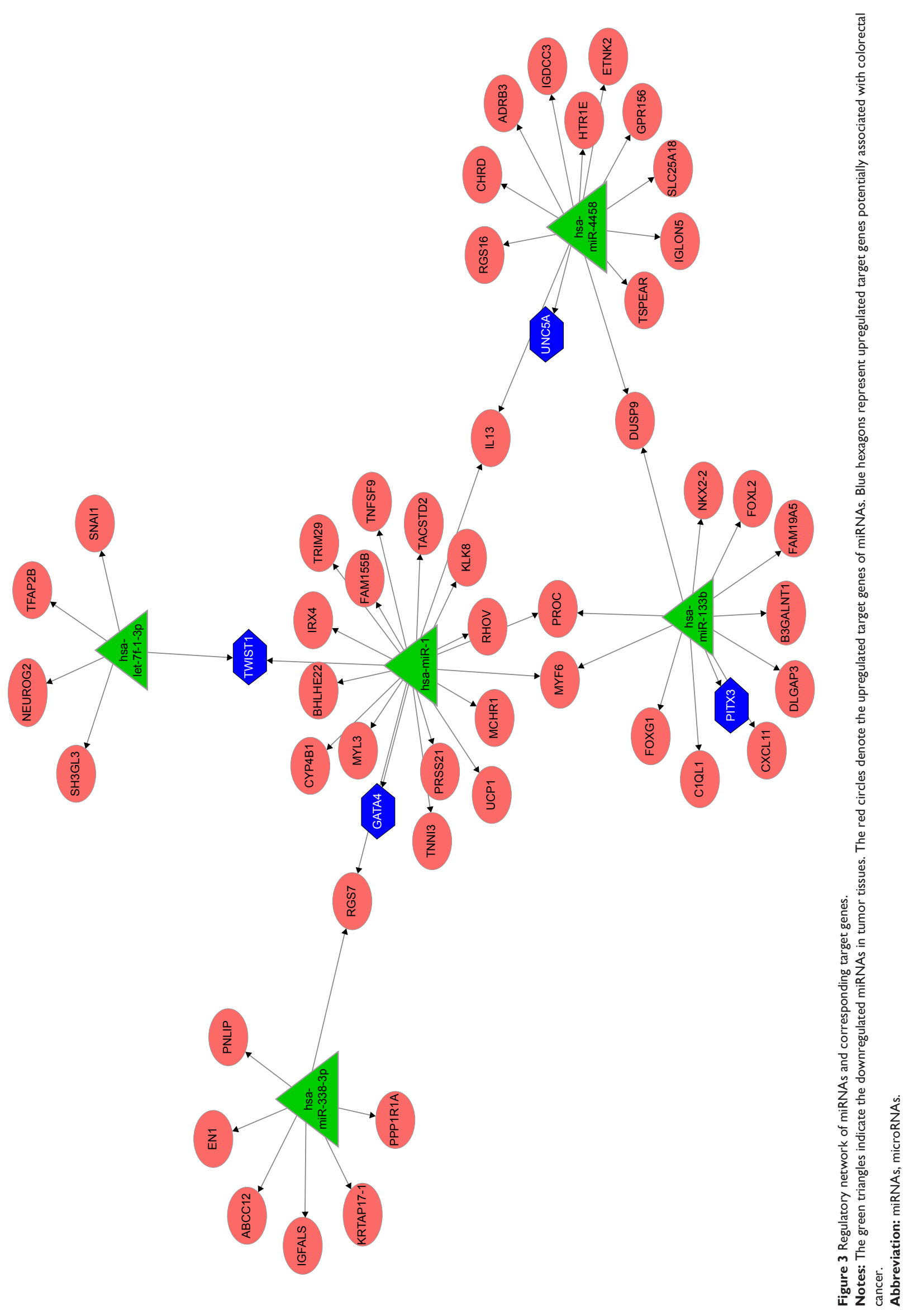


gene of miRNA-450b-3p, whose expression level showed a downward trend in CRC. The CEA family genes have been revealed to encode a group of intercellular adhesion molecules ${ }^{47,48}$ and to promote metastasis of human CRC..$^{49}$ CEACAM7, a member of CEA family, has been demonstrated to be downregulated in the transgenics' colons $^{50}$ and in human $\mathrm{CRC},{ }^{48}$ which may function by suppressing CRC growth. ${ }^{51}$ Based on the previous researches, we deduced that the overexpressed miRNA-450b-3p might act as oncogene by downregulating $C E A C A M 7$ and could be suggested as a novel target to treat advanced CRC.

\section{Conclusion}

The screened miRNAs, especially miRNA-1 and miRNA-let7f-1-3p targeting TWIST1, miRNA-133b and miRNA-4458 targeting DUSP9, as well as miRNA-450b-3p targeting CEACAM7, might function as tumor suppressors or oncogenes in CRC development. Those miRNAs could be suggested as potential therapeutic targets. However, their involvement in $\mathrm{CRC}$ was determined from bioinformatics prospective. In future works, selected miRNAs and DEGs will be validated using real-time PCR and Western blotting.

\section{Disclosure}

The authors report no conflicts of interest in this work.

\section{References}

1. Stewart BW, Wild C. World Cancer Report 2014. Geneva: World Health Organization; 2014.

2. O'Connell JB, Maggard MA, Ko CY. Colon cancer survival rates with the new American Joint Committee on Cancer sixth edition staging. J Natl Cancer Inst. 2004;96(19):1420-1425.

3. Kloosterman WP, Plasterk RH. The diverse functions of microRNAs in animal development and disease. Dev Cell. 2006;11(4):441-450.

4. Croce CM. Causes and consequences of microRNA dysregulation in cancer. Nat Rev Genet. 2009;10(10):704-714.

5. Calin GA, Croce CM. MicroRNA signatures in human cancers. Nat Rev Cancer. 2006;6(11):857-866.

6. Volinia S, Calin GA, Liu CG, et al. A microRNA expression signature of human solid tumors defines cancer gene targets. Proc Natl Acad Sci US A. 2006;103(7):2257-2261.

7. Nielsen BS, Jørgensen S, Fog JU, et al. High levels of microRNA-21 in the stroma of colorectal cancers predict short disease-free survival in stage II colon cancer patients. Clin Exp Metastasis. 2011;28(1):27-38.

8. Li T, Leong MH, Harms B, Kennedy G, Chen L. MicroRNA-21 as a potential colon and rectal cancer biomarker. World J Gastroenterol. 2013;19(34):5615-5621

9. Akao Y, Nakagawa Y, Naoe T. Let-7 microRNA functions as a potential growth suppressor in human colon cancer cells. Biol Pharm Bull. 2006;29(5):903-906.

10. Gregersen LH, Jacobsen AB, Frankel LB, Wen J, Krogh A, Lund AH. MicroRNA-145 targets yes and stat 1 in colon cancer cells. PLoS One. 2010;5(1):e8836.

11. Jin Y, Zhao M, Xie Q, Zhang H, Wang Q, Ma Q. MicroRNA-338-3p functions as tumor suppressor in breast cancer by targeting sox 4 . Int J Oncol. 2015;47(4):1594-1602.
12. Tazawa H, Tsuchiya N, Izumiya M, Nakagama H. Tumor-suppressive miR-34a induces senescence-like growth arrest through modulation of the E2F pathway in human colon cancer cells. Proc Natl Acad Sci US A. 2007;104(39):15472-15477.

13. Li J, Liu Y, Wang C, et al. Serum miRNA expression profile as a prognostic biomarker of stage II/III colorectal adenocarcinoma. Sci Rep. 2015; 5:12921.

14. Gopalakrishnan C, Kamaraj B, Purohit R. Mutations in microRNA binding sites of CEP genes involved in cancer. Cell Biochem Biophys. 2014;70(3):1933-1942.

15. Kamaraj B, Gopalakrishnan C, Purohit R. In silico analysis of miRNAmediated gene regulation in OCA and OA genes. Cell Biochem Biophys. 2014;70(3):1923-1932.

16. Bhaumik P, Gopalakrishnan C, Kamaraj B, Purohit R. Single nucleotide polymorphisms in microRNA binding sites: Implications in colorectal cancer. ScientificWorldJournal. 2014;2014:547154.

17. Kumar A, Rajendran V, Sethumadhavan R, Purohit R. Evidence of colorectal cancer-associated mutation in MCAK: a computational report. Cell Biochem Biophys. 2013;67(3):837-851.

18. Zhang Y, Talmon G, Wang J. MicroRNA-587 antagonizes 5-FU-induced apoptosis and confers drug resistance by regulating PPP2R1B expression in colorectal cancer. Cell Death Dis. 2015;6:e1845.

19. Muller S, Raulefs S, Bruns $P$, et al. Next-generation sequencing reveals novel differentially regulated mRNAs, IncRNAs, miRNAs, sdRNAs and a piRNA in pancreatic cancer. Mol Cancer. 2015;14:94.

20. Röhr C, Kerick M, Fischer A, et al. High-throughput miRNA and mRNA sequencing of paired colorectal normal, tumor and metastasis tissues and bioinformatic modeling of miRNA-1 therapeutic applications. PLoS One. 2013;8(7):e67461.

21. Barrett T, Wilhite SE, Ledoux P, et al. NCBI GEO: Archive for functional genomics data sets - update. Nucleic Acids Res. 2013;41:D991-D995.

22. FastQC [webpage on the Internet]. Babraham: Babraham Bioinformatics; 2014. Available from: http://www.bioinformatics.bbsrc.ac.uk/ projects/fastqc/. Accessed November 13, 2015.

23. Burge SW, Daub J, Eberhardt R, et al. Rfam 11.0: 10 years of RNA families. Nucleic Acids Res. 2013;41:D226-D232.

24. Langmead B, Trapnell C, Pop M, Salzberg SL. Ultrafast and memoryefficient alignment of short DNA sequences to the human genome. Genome Biol. 2009;10(3):R25.

25. Trapnell C, Pachter L, Salzberg SL. TopHat: discovering splice junctions with RNA-seq. Bioinformatics. 2009;25(9):1105-1111.

26. Kozomara A, Griffiths-Jones S. miRBase: Integrating microRNA annotation and deep-sequencing data. Nucleic Acids Res. 2011;39: D152-D157.

27. Friedlander MR, Mackowiak SD, Li N, Chen W, Rajewsky N. miRDeep2 accurately identifies known and hundreds of novel microRNA genes in seven animal clades. Nucleic Acids Res. 2012;40(1):37-52.

28. Trapnell C, Williams BA, Pertea G, et al. Transcript assembly and quantification by RNA-seq reveals unannotated transcripts and isoform switching during cell differentiation. Nat Biotechnol. 2010;28(5):511-515.

29. Tarazona S, Garcia-Alcalde F, Dopazo J, Ferrer A, Conesa A. Differential expression in RNA-seq: a matter of depth. Genome Res. 2011;21(12): 2213-2223.

30. Lewis BP, Burge CB, Bartel DP. Conserved seed pairing, often flanked by adenosines, indicates that thousands of human genes are microRNA targets. Cell. 2005;120(1):15-20.

31. Dweep H, Sticht C, Pandey P, Gretz N. miRWalk-database: prediction of possible miRNA binding sites by "walking" the genes of three genomes. J Biomed Inform. 2011;44(5):839-847.

32. Xiao F, Zuo Z, Cai G, Kang S, Gao X, Li T. miRecords: an integrated resource for microRNA-target interactions. Nucleic Acids Res. 2009;37: D105-D110.

33. Zhao M, Sun J, Zhao Z. TSGene: a web resource for tumor suppressor genes. Nucleic Acids Res. 2013;41:D970-D976.

34. Chen JS, Hung WS, Chan HH, Tsai SJ, Sun HS. In silico identification of oncogenic potential of fyn-related kinase in hepatocellular carcinoma. Bioinformatics. 2013;29(4):420-427. 
35. Wingender E, Dietze P, Karas H, Knuppel R. TRANSFAC: a database on transcription factors and their DNA binding sites. Nucleic Acids Res. 1996;24(1):238-241.

36. Reid JF, Sokolova V, Zoni E, et al. miRNA profiling in colorectal cancer highlights mir-1 involvement in met-dependent proliferation. Mol Cancer Res. 2012;10(4):504-515.

37. Scheel C, Weinberg RA. Cancer stem cells and epithelial-mesenchymal transition: concepts and molecular links. Semin Cancer Biol. 2012; 22(5-6):396-403.

38. Yang G, Yuan J, Li K. EMT transcription factors: implication in osteosarcoma. Med Oncol. 2013;30:1-5.

39. Jaiswal AS, Marlow BP, Gupta N, Narayan S. Beta-catenin-mediated transactivation and cell-cell adhesion pathways are important in curcumin (diferuylmethane)-induced growth arrest and apoptosis in colon cancer cells. Oncogene. 2002;21(55):8414-8427.

40. Valdés-Mora F, del Pulgar TG, Bandrés E, et al. TWIST1 overexpression is associated with nodal invasion and male sex in primary colorectal cancer. Ann Surg Oncol. 2009;16(1):78-87.

41. Ichimi T, Enokida H, Okuno Y, et al. Identification of novel microRNA targets based on microRNA signatures in bladder cancer. Int $J$ Cancer. 2009;125(2):345-352.

42. Kano M, Seki N, Kikkawa N, et al. Mir-145, mir-133a and mir-133b: tumor-suppressive miRNAs target FSCN1 in esophageal squamous cell carcinoma. Int J Cancer. 2010;127(12):2804-2814.

43. Bhaduri A, Sowdhamini R. A genome-wide survey of human tyrosine phosphatases. Protein Eng. 2003;16(12):881-888.

44. Owens DM, Keyse SM. Differential regulation of MAP kinase signalling by dual-specificity protein phosphatases. Oncogene. 2007;26(22): 3203-3213.
45. Xu T, Zhou M, Peng L, et al. Upregulation of CD147 promotes cell invasion, epithelial-to-mesenchymal transition and activates MAPK/ERK signaling pathway in colorectal cancer. Int J Clin Exp Pathol. 2014; 7(11):7432-7441.

46. Sansom OJ, Meniel V, Wilkins JA, et al. Loss of Apc allows phenotypic manifestation of the transforming properties of an endogenous K-ras oncogene in vivo. Proc Natl Acad Sci U S A. 2006;103(38): 14122-14127.

47. Stanners CP. Cell Adhesion and Communication Mediated by the CEA Family. Boca Raton, FL: CRC Press; 2003.

48. Hammarstrom S, Olsen A, Teglund S, Baranov V. The nature and expression of the human cea family. In: Cell Adhesion and Communications Mediated by the CEA Family Basic and Clinical Perspectives, Volume 5. Amsterdam: Harwood Academic; 2003:1-30.

49. Hashino J, Fukuda Y, Oikawa S, Nakazato H, Nakanishi T. Metastatic potential of human colorectal carcinoma SW1222 cells transfected with cDNA encoding carcinoembryonic antigen. Clin Exp Metastasis. 1994;12(4):324-328.

50. Chan $\mathrm{CH}$, Stanners CP. Novel mouse model for carcinoembryonic antigen-based therapy. Mol Ther. 2004;9(6):775-785.

51. Schölzel S, Zimmermann W, Schwarzkopf G, Grunert F, Rogaczewski B, Thompson J. Carcinoembryonic antigen family members ceacam 6 and ceacam 7 are differentially expressed in normal tissues and oppositely deregulated in hyperplastic colorectal polyps and early adenomas. Am J Pathol. 2000;156(2):595-605.
OncoTargets and Therapy

\section{Publish your work in this journal}

OncoTargets and Therapy is an international, peer-reviewed, open access journal focusing on the pathological basis of all cancers, potential targets for therapy and treatment protocols employed to improve the management of cancer patients. The journal also focuses on the impact of management programs and new therapeutic agents and protocols on

\section{Dovepress}

patient perspectives such as quality of life, adherence and satisfaction. The manuscript management system is completely online and includes a very quick and fair peer-review system, which is all easy to use. Visit http://www.dovepress.com/testimonials.php to read real quotes from published authors. 